\title{
Aprender lengua y cultura mapuche en la escuela: Estudio de caso de la implementación del nuevo Sector de Aprendizaje Lengua Indígena desde un análisis de "recursos educativos"*
}

\author{
Learning Mapuche Language and Culture at School: Case Study of the Implementation \\ of the New Subject Indigenous Language in the light of an Analysis of \\ "Educational Resources"
}

\author{
Aprender língua e cultura mapuche na escola: Estudo de caso da implantacäo de novo \\ Setor Língua Indígena desde uma análise de "recursos educativos"
}

\author{
Laura Luna, Pelayo Benavides, Pablo Gutiérrez, Manuela Alchao, Alberto Dittborn. ${ }^{a}$ \\ a Pontificia Universidad Católica de Chile, Campus Villarrica. Fono: 56-045-2-411667. Correos electrónicos: \\ 1luna@uc.cl, sbenavme@uc.cl,pgutieri@uc.cl, manualchao@gmail.com, adittbob@gmail.com
}

\section{RESUMEN}

El artículo analiza un caso de implementación del Sector de Aprendizaje Lengua Indígena focalizándose en los recursos educativos empleados, en cuanto objetos "mediadores" del aprendizaje. Con base en el trabajo etnográfico de observación participante en clases, se examina el desenvolvimiento de las actividades didácticas poniendo el foco de atención en los recursos o materiales utilizados, incluyendo los que fueron introducidos por iniciativa de los mismos investigadores en el marco de un proceso de búsqueda de estrategias didácticas efectivas de parte de la profesora del Sector y de una solicitud de apoyo de parte de la escuela. Junto con introducir una definición técnica de recurso educativo, se analizan las diferentes situaciones de aprendizaje desde la perspectiva de la "cognición situada" que permite identificar los componentes que convierten especialmente a las actividades más "lúdicas" no sólo en intrínsecamente motivadoras, sino también en estrategias efectivas para el desarrollo de habilidades académicas.

Palabras clave: educación intercultural, recursos educativos, juego, aprendizaje situado.

\begin{abstract}
The article analyzes a case of implementation of the curricular subject Indigenous Language focusing on the educational resources employed as "learning mediators". Based on an ethnographic work of participant observation in class, the development of teaching and learning activities is examined, giving attention to the educational resources or materials employed. These included those intentionally introduced by the researchers who collaborated with the teacher in seeking better teaching strategies, and the explicit school demand for support. Besides introducing a technical definition of educational resource, different learning situations are analyzed from the perspective of "situated cognition" which allows identifying those elements that make "playful activities" in particular, not only intrinsically motivating but also effective for the development of academic abilities.
\end{abstract}

Key words: intercultural education, educational resources, game, situated learning.

\section{RESUMO}

Analisa-se um caso de implantação do setor de Aprendizagem Língua Indígena focalizando-se nos recursos educativos empregados enquanto objetos "mediadores" da aprendizagem. Com base no trabalho etnográfico de

* Fondo Interno 2011, Pontificia Universidad Católica de Chile, Campus Villarrica. 
observação participante em classes, examina-se o desenvolvimento das atividades didáticas, focando a atenção nos recursos e materiais utilizados, incluindo aqueles introduzidos por investigadores em um marco de um processo de busca de estratégias didáticas efetivas por parte da professora do Setor e da solicitação de apoio por parte da escola. Junto com a introdução de uma definição técnica de recursos educativos, analisam-se diferentes situações do aprendizado desde uma perspectiva da "cognição situada", o que permite identificar os componentes que convertem especialmente em as atividades mais lúdicas não só intrinsecamente motivadoras como também em estratégias efetivas para o desenvolvimento de habilidades acadêmicas.

Palavras chave: educação intercultural, recursos educativos, jogo, aprendizagem situada.

\section{INTRODUCCIÓN}

Este trabajo se basa en el estudio de la implementación de un reciente componente de la política de educación intercultural bilingüe en Chile, es decir, la entrada en vigencia del nuevo Sector de Aprendizaje Lengua Indígena en el currículo nacional, desde marzo de 2010. A partir de esta fecha el Sector Lengua Indígena (SLI), según lo establecido por el Decreto N. 280 sobre Lenguaje y Comunicación y en el marco de la Ley General de Educación de 2009 (LEGE), se ofrece obligatoriamente en los establecimientos que tengan por lo menos el $50 \%$ de estudiantes indígenas, siendo voluntario en esos sectores de alta densidad indígena o en otras áreas donde se quiera promover el valor de la interculturalidad y bilingüismo. A partir del 2013, también los establecimientos que cuentan con el $20 \%$ de niños indígenas están obligados a impartirlo. Empezando con el primero básico e incorporando cada año un nuevo nivel, el Decreto apunta a cubrir la necesidad de promover el uso y la conservación de los idiomas indígenas en toda la educación básica.

La introducción del SLI representa la intervención más significativa, en el marco de la política educacional nacional, para el respeto de los derechos a la educación de los pueblos indígenas, y en particular, del derecho a una educación conforme a la identidad y cultura de los niños y niñas, tal como está establecido en documentos internacionales como la Convención del Niño del 1989, el Convenio 169 de la OIT del 1989 (ratificado en Chile en 2008), y la Declaración de las Naciones Unidas sobre los Derechos de los Pueblos Indígenas de 2007. Pese a los límites que aún presenta la propuesta del SLI, como su carácter optativo y voluntario (para establecimientos con baja densidad indígena), y el mínimo de $20 \%$ de estudiantes indígenas para su ofrecimiento, su introducción representa un avance substancial en la promoción del bilingüismo y de los derechos culturales de los pueblos indígenas, especialmente porque la enseñanza de la lengua y la cultura indígena, al incorporarse en el marco curricular nacional, adquiere una nueva legitimación (Loncon, 2010), disminuye el riesgo de "desmenuzamiento" y decontextualización de la cultura común en la práctica docente, y evita a los docentes la difícil tarea de integrar los contenidos de la cultura indígena con los objetivos fundamentales y los contenidos mínimos (Luna e Hirmas, 2005).

El SLI, contemplando la flexibilidad según el contexto lingüístico y cultural local, apunta al desarrollo de la Oralidad y de la Comunicación Escrita. La Oralidad está conformada por dos aspectos: Tradición Oral y Comunicación Oral. La primera se refiere a "prácticas lingüísticas asociadas a la cultura, que forman parte del patrimonio histórico de cada pueblo" como relatos, mitos, canciones tradicionales, momentos rituales (MINEDUC, 2009: en línea). En cambio, la segunda "se concibe como interacciones cotidianas, prácticas discursivas y conocimiento y manejo de la lengua” (p. ej.: formas de 
saludo y de convivencia) (MINEDUC, 2009: en línea). Por otra parte, el desarrollo de la Comunicación Escrita se refiere a la capacidad de leer y escribir palabras de uso cotidiano en lengua indígena, y de identificar distintos tipos de textos en lengua indígena y mostrar interés por la lectura de éstos.

En su diseño, el SLI contempla una "dupla pedagógica" a cargo de implementarlo, compuesta por el profesor mentor y por un educador tradicional, quien debiera ser normalmente un miembro de la comunidad local, conocedor de su cultura y de su variante lingüística. Estas dos figuras se complementarían en el trabajo de aula, al tener el educador los conocimientos culturales, y el profesor los conocimientos pedagógicos necesarios para transmitirlos a los niños. También el Programa ofrece asesoría y materiales didácticos de apoyo para la dupla, siempre que el establecimiento que imparte el Sector esté adscrito al Programa. En la realidad, tal como se ha constatado (MINEDUC, 2012: 32), muchos establecimientos no están adscritos al Programa pero igualmente imparten el Sector por medio de los recursos de la Subvención Escolar Preferencial (Ley SEP).

La escuela de la comuna de Pucón que ha sido objeto de este estudio pertenece a este grupo. Con recursos SEP se financiaba la contratación de una profesora por horas dedicada exclusivamente a la enseñanza del SLI en primero y segundo básico (en el año 2011, cuando se realizó el estudio). El caso de esta escuela difiere también de otros en los que se imparte el SLI porque la profesora no trabajaba en dupla, es decir, con el apoyo de un educador. Se estimaba que, siendo egresada de la formación pedagógica en Educación Intercultural Bilingüe (EIB) de la Universidad Católica de Temuco, y perteneciendo a una de las comunidades indígenas de la comuna de Pucón, tuviera ambos tipos de conocimientos necesarios para impartir el SLI, el cultural y el pedagógico.

Sin embargo, la profesora presentaba dificultades en los dos ámbitos, no teniendo el mapudungun como lengua materna, por un lado, y no contando con una larga experiencia de enseñanza escolar, por el otro. No obstante, su actitud reflexiva y autocrítica, su inquietud por mejorar sus prácticas, su afán por buscar nuevas formas de enseñar y su capacidad de atreverse a implementar nuevos métodos y estrategias didácticas le conferían una ventaja comparativa respecto a muchos otros docentes. En este sentido, el perfil de esta docente era muy apropiado para el objetivo de nuestro estudio, que era examinar las prácticas docentes en el SLI focalizando la mirada en los recursos implementados. La variedad de prácticas y de recursos adoptados por la profesora en su búsqueda de nuevas estrategias ofrecieron a los investigadores, en un arco de tiempo limitado, un amplio abanico de "casos" a analizar.

La mirada en profundidad que permite el método etnográfico aplicado al aula escolar, nos ha entregado un rico material de registro de las prácticas docentes durante las clases del SLI y de su impacto en los niños. El objetivo de este trabajo es examinarlo a la luz de la perspectiva de la "cognición situada" (Rogoff, 1984), y de una visión de recursos educativos como "artefactos mediadores" (Acosta-Iriqui y Esteban, 2010; Holland \& Valsiner, 1988). La idea de recurso educativo "óptimo" que ha construido este equipo multidisciplinario se articula con estas perspectivas teóricas.

Concebido con un objetivo etnográfico y exploratorio de las prácticas sociales y pedagógicas que caracterizaban la implementación del SLI en la escuela de Pucón, el presente estudio fue, en la marcha, acotando y a la vez ampliando su alcance. Por un lado, la mirada hacia las prácticas docentes se concentró en los recursos educativos empleados y, por otro, llegó a abarcar también los recursos diseñados por un equipo de profesionales convocado por los investigadores e introducidos en las dinámicas de clase. El objetivo, la 
implementación, el impacto de tales recursos también se convirtió en objeto de descripción etnográfica y de análisis. La modificación del foco de estudio obedeció, en primer lugar, a la relevancia atribuida a la mediación educativa y los objetos implicados a partir del marco teórico del estudio; en segundo lugar, a la petición expresa por la profesora participante en el estudio de contar con apoyo en la creación de recursos educativos pertinentes. Tratándose de un contexto de intervención social altamente sensible, como es el campo educativo, se consideró necesario, por razones éticas, acoger la demanda de una actitud más "proactiva" de parte de los investigadores (Luna, 2012) y abandonar el carácter exclusivamente investigativo del estudio para acercarlo más a un modelo de investigación-acción.

En este marco, el registro etnográfico se centró en las acciones, interacciones y reacciones de los sujetos conectadas con la utilización de los recursos educativos presentados (en particular, concretos). Así, el objetivo del artículo es presentar una descripción y análisis de los materiales utilizados en la clase del Sector de Lengua y Cultura impartida en primer y segundo básico durante el año escolar 2011. Los materiales aludidos son abordados como mediadores del aprendizaje y de la participación de los niños, según el enfoque sociocultural y la visión vigostkiana. Dicho enfoque reconoce el papel desplegado por artefactos de diversa índole como mediadores de la cognición, además de asumir al aprendizaje como un fenómeno "situado". Esta idea amplía los límites del aprendizaje hasta aspectos que van más allá de lo intencionado por los agentes educadores y los objetivos institucionales de enseñanza, revalorizando el punto de vista del aprendiz como agente dentro del mismo proceso.

\section{EL DESARROLLO HUMANO, LOS ARTEFACTOS Y EL CONTEXTO SOCIO- CULTURAL}

El énfasis en los recursos se enmarca en el llamado "enfoque sociocultural" o "históricocultural” (Esteban y Ratner, 2010: 122; Rogoff, 2003: 10), según el cual el ser humano es visto como un "agente activo" de su desarrollo, como un constructor de los contextos en donde se desempeña, enfatizando la retroalimentación presente en las influencias de sistemas sociales más amplios (Markus \& Hamedani, 2007).

Rogoff (2003: 237) afirma que el desarrollo cognitivo, en este marco de referencia "sociocultural", no se entiende como la mera adquisición receptiva de conocimientos y habilidades. El desarrollo cognitivo pasa a ser definido entonces como el cambio en las maneras de percibir, clasificar, reflexionar acerca de, recordar y entender el mundo (entre otras actividades) por parte de los sujetos. Sin embargo, un aspecto central de dichas actividades es el hecho de que son actividades compartidas con otros sujetos, en prácticas culturales y tradiciones de comunidades dadas. En la mayoría de los casos, estas actividades son "mediadas" por herramientas culturales que se distribuyen en un rango amplio que va desde el polo abstracto (elementos numéricos, fórmulas lógicas, etc.) al más concreto (objetos).

A partir de la toma de conciencia sobre la importancia de la mediación y de los contextos socioculturales e históricos en los cuales se desarrollan los sujetos de manera bidireccional, surge la necesidad de analizar otros elementos que están presentes en dichos contextos, además de sujetos y comunidades. Tal como postula Shweder (1990, Cit. en Markus \& Hamedani, 2007: 6), el sujeto intencional establece una interdependencia con un mundo intencional, es decir, con un mundo de significados. Este mundo es, consecuentemente, un 
mundo humano "artefactual", poblado por productos de nuestro propio diseño, cargados de significados y/o potencialmente utilizables para vehiculizar significados.

De esta manera, el énfasis del enfoque sociocultural en el uso de instrumentos/artefactos psicológicos o culturales, tales como mapas, diagramas o letras, o aquellos denominados "tecnológicos", como computadores, herramientas mecánicas o libros, da cabida al análisis y estudio cultural del desarrollo humano. Este último es visto, por tanto, en estrecha relación con "el efecto psicológico que tiene la apropiación, el uso y manejo de determinados artefactos de origen y transmisión sociocultural" (Esteban y Ratner, 2010: 123).

Desde la perspectiva de Vygotsky, tanto las herramientas técnicas como las psicológicas (el lenguaje) son productos históricos que forman parte de los sistemas de significación colectiva. Es decir, su utilización se basa en los acuerdos previos y los usos que se han aprendido en el curso de la interacción social con otros. De esta manera, objetos que son encontrados en un primer momento en una serie de intercambios sociales evolucionan desde ser simplemente elementos constituyentes de rutinas interaccionales, a símbolos que son incorporados en procesos complejos interpersonales (Holland \& Valsiner, 1988: 250, 252). Por otra parte, las personas interpretan los contextos y el uso que se da a los artefactos mediadores en aquellos, utilizando como referencia los correspondientes modelos culturales que han aprendido en sus medios sociales (Holland \& Valsiner, 1988: 257).

De esta manera, como afirman Esteban y Ratner, es posible ver las relaciones que se sostienen entre los procesos psicológicos superiores y la actividad de cada sujeto en contextos socioculturales dados. Los primeros se caracterizan por su "origen social y cultural, ya que en forma de productos colectivos forman parte de las habilidades, destrezas, conocimientos de un determinado grupo humano" (2010: 124). Sin embargo, la actividad de los sujetos estaría siempre siendo mediada culturalmente, revelando la conexión entre el funcionamiento del individuo y los instrumentos o artefactos culturales que permiten dicho funcionamiento. Esto último implicaría que no resulta posible "separar la cognición de los instrumentos culturales que la sustentan y la conforman".

De acuerdo al marco de referencia expuesto, por tanto, hay una ilimitada cantidad de elementos que pueden ser utilizados como artefactos mediadores de procesos cognitivos, hasta incluir prácticas sociales o creencias, tal como plantean Acosta-Iriqui y Esteban (2010). Sin embargo, es oportuno aclarar que utilizar algo como artefacto-recurso mediador del pensamiento, según la definición amplia que hacen estos autores, no equivale del todo a tener recursos educativos en el sentido empleado en nuestro análisis. Si bien ambos conceptos se traslapan y comparten características, hay otros elementos que los diferencian y que es necesario especificar.

\section{RECURSOS EDUCATIVOS EN LA CLASE DEL SECTOR LENGUA INDÍGENA}

"Recurso educativo" es un término presente en la universalidad de los contextos escolares y en el lenguaje de los docentes, siendo un mediador entre el sujeto y el objeto de su conocimiento en un contexto cultural determinado. A pesar de su amplia difusión y utilización, el concepto padece de una multiplicidad de interpretaciones, especificaciones y términos que por separado reducen su comprensión. Dittborn et al. (2008) se enfrentaron a este problema a la hora de especificar y analizar los "recursos educativos" como medios o materiales de enseñanza. Sólo para esta acepción aparecen una diversidad de términos como: 
"medios auxiliares", "recursos didácticos", "ayudas didácticas", "materiales curriculares", "nuevas tecnologías" o "nuevos canales de comunicación", entre otros. Si bien cada una de ellas pareciera apuntar a una definición similar, en sus especificaciones se pueden aislar categorías contrapuestas, que no aportan a lograr que el concepto sea consistente.

Para graficar esta situación se muestran algunas de las diferencias en los criterios de definición: uso dentro o fuera del aula (Szendrei, 1996; Zabala y Arnau, 2007), presencia $o$ ausencia de la intencionalidad didáctica en la creación o utilización de un recurso (Bartolomé, 2000; Coriat, 1997), uso en procesos de enseñanza y aprendizaje (Carrasco y Basterretche, 1998; Gimeno Sacristán, 1990; Good, 1973; Ossana, Bargellini y Laurino, 1984), o uso en el proceso de mediación o ajeno a éste (Bartolomé, 2000; Corrales y Sierras, 2012).

Desde nuestra perspectiva, el recurso educativo es un medio que materializa el diseño de una experiencia, que busca facilitar procesos de enseñanza-aprendizaje y el logro de objetivos transversales. Esta experiencia puede llegar a ser "óptima"l si contempla el uso de más de un canal de percepción e impacta la mayor cantidad posible de sentidos, promueve mayoritariamente la resolución de situaciones-problemas mediante el trabajo colaborativo entre pares, y trabaja contenidos o evoca situaciones que se apoyan en entornos cercanos al niño. Esta noción de recurso "ideal" o efectivo está plasmada por una visión de la cognición y del aprendizaje como "situado" (Rogoff, 1984), en el cual el contexto sociocultural, las interacciones y un desafío de orden pragmático juegan un papel determinante. Esta idea de recurso educativo ha orientado el diseño de los dos tipos de materiales que se introdujeron, de manera exploratoria, en las clases del Sector, concretándose especialmente por las características que se discuten más adelante, en el juego del rakin awar.

En línea con esta concepción de recurso y de aprendizaje, hemos abordado el análisis de los materiales y medios desplegados en el Sector, desde una mirada que identifica en un primer nivel el grado de participación de los niños en cuanto principales usuarios de esos recursos. Es decir, hemos clasificado los tipos de recursos empleados en las clases del Sector, incluso los que fueron introducidos por los investigadores, en función de su utilización o "manipulación" predominante: de parte de la profesora o de parte de los niños.

El primer grupo de recursos, en que se cuentan los "epew", las "canciones sobre partes del cuerpo" y los "segmentos de tiempo y espacio", aúna materiales, objetos y medios que la profesora utilizó principalmente como un soporte visual para explicar o exponer determinados contenidos: personajes y situaciones de los cuentos tradicionales mapuche, las fases del ciclo del sol durante el día, y las actividades asociadas en la vida rural mapuche. Por otra parte, las canciones también presentan la peculiaridad de ser un recurso empleado por la profesora para transmitir ciertos contenidos de manera más eficaz -por medio de elementos como el ritmo y la rima-. Se requiere, sin embargo, que los niños aprendan a manejarlo en forma autónoma para que éste pueda cumplir su objetivo de aprendizaje, es decir, que puedan reproducir la canción y pronunciar oportunamente los conceptos en mapudungun.

La "experiencia óptima" es una expresión acuñada por Mihaly Csikszentmihalyi (1998) y se basa en el concepto de flujo, el cual se ha transformado en una de las mayores aportaciones al estudio de la motivación intrínseca que se ha producido en los últimos años. Este concepto hace referencia a situaciones altamente positivas, a experiencias autotélicas y a la satisfacción que genera la realización de alguna actividad. Las principales características fenomenológicas que definen la situación de flujo son: una situación de reto o desafío, focalización de la atención en la acción, metas claras, retroalimentación sobre la acción, sentimientos de control y despreocupación sobre uno mismo. 
En el segundo grupo, que reúne "fichas", el "rompecabezas" y el "rakin awar" encontramos, en cambio, una variedad de artefactos y materiales diseñados para uso exclusivo de los niños, para llevar a cumplimiento la actividad asignada. La relación entre la actividad realizada por los niños y el medio es, contrariamente a los materiales del primer grupo, de total interdependencia; no son un mero soporte a la actividad didáctica (p. ej.: a una presentación) sino el objeto mismo de ésta. La actividad no podría en ningún caso desarrollarse sin dichos artefactos, y éstos se convierten en mediadores significativos sólo en el marco de ésta. Si bien todo artefacto puede convertirse en un mediador del pensamiento y, dependiendo del contexto y del "uso", ser más o menos significativo como vehículo de construcciones simbólicas, los materiales del segundo grupo requieren, en comparación con los del primer grupo, un alto grado de manipulación. Ésta es crucial para lograr incluso un mínimo nivel de "significatividad" del recurso. En otras palabras, el carácter de herramientas de los artefactos del segundo grupo es inequívoco. Éste es uno de los aspectos centrales que comparten tres tipos de recursos muy diferentes unos de otros.

De todos los recursos mencionados solamente los epew, apoyados en imágenes desde el proyector y las fichas, eran parte de la práctica habitual de la docente, mientras los otros asumieron un carácter más bien experimental: la profesora los empleó en ocasiones puntuales, tanto en primero como en segundo año, en el marco de un proceso de búsqueda propia de soluciones innovadoras para aumentar la efectividad de sus clases.

Al respecto, es oportuno señalar que ha sido posible notar, a lo largo del período de observación de clases (un año escolar), una tendencia general de parte de la profesora a buscar e implementar recursos que implicaran una participación más directa de parte de los niños. Por lo mismo, durante el segundo semestre, se encontraron con más frecuencia en sus clases actividades que la profesora denomina "lúdicas", más parecidas a juegos y altamente motivadoras para los niños por el papel más protagónico que ellos adquirieron en éstas. Por otra parte, no se observaron presentaciones expositivas en formato power point durante los últimos meses del año. Nos parece una hipótesis fundada que este cambio en la práctica de la profesora se deba además que a su inquietud por generar más motivación en sus alumnos, a la interacción con el equipo de investigadores y, en particular, con los diseñadores quienes lideraron las conversaciones sobre las posibles formas para promover la motivación y participación en los niños.

\subsection{RECURSOS UTILIZADOS POR LA DOCENTE}

\subsubsection{Los epew}

"Un día estaba la machi buscando remedios en el campo" -empieza la profesora-. De pronto se establece un silencio absoluto en la clase y todos los niños ponen atención a la historia de la machi y del zorro que la profesora cuenta por medio de imágenes proyectadas en la pared. Se trata de representaciones cercanas a las ilustraciones de libros infantiles, por lo tanto, resultan amenas y atractivas para un público joven. “QQué pájaro es ese?”pregunta la profesora-, "Tiuque" contestan los niños. La profesora cuenta la historia con un tono animado y teatral que mantiene la atención de los alumnos. Éstos de vez en cuando intervienen con una palabra respondiendo una pregunta o completando una frase de la profesora: "Le dice el zorro a la machi: Yo te voy a pagar con mi [...]” "¡Manta!" agregan los niños ayudados por las imágenes. "Muy bien”, refuerza la profesora. 
Los epew son muy eficaces en captar y mantener la atención de los niños. Los personajes son de particular interés especialmente si se trata de animales que los niños conocen por vivir en el campo: puma, zorro, treile, tiuque, etc. La profesora enriquece la presentación de los personajes con comentarios que vienen de su propia experiencia: "los treiles que están empollando se ponen nerviosos cuando las personas pasan cerca de los huevos porque algunos niños los toman para romperlos". Los niños también espontáneamente cuentan experiencias personales relacionadas con esos animales: "Profe, sabe que yo me escondo en los árboles cuando veo el zorro"; "yo lo mato porque salgo con la escopeta de mi papá para ir a matar", "Yo maté un zorro con alambre de púa".

El objetivo principal del epew pareciera ser una reflexión moral sobre el comportamiento de los personajes: “¿Cuál es la enseñanza?”, pregunta la docente al final de la historia. "No hay que pelear", dice una niña. Los conceptos de weza (malo) y küme (bueno) que aluden a cualidades morales son reforzados durante la ejecución de la tarea escrita que sigue al epew, en la cual los niños tienen que pintar y recortar los animales representados en las fichas que les han sido entregadas. Los niños expresan un cierto descontento cuando la profesora les anuncia, al término del cuento, que van a "trabajar". Algunos piden más epew.

\subsubsection{Las canciones sobre partes del cuerpo}

Si bien la utilización de canciones, como recurso educativo, se pudo observar en ocasiones sólo en términos receptivos de escucha con utilización de equipos, también se evidenció de manera directa y participativa. Uno de los casos concretos observados fue en referencia al tema de las partes del cuerpo - che kalul- y sus nombres en mapudungun. La profesora les pide a todos que se pongan de pie para cantar una canción que trata sobre el cuerpo, explicando que incluye mímica y que necesitarán moverse.

De esta forma, ella va cantando y modelando al mismo tiempo los movimientos necesarios que acompañaban la letra de la canción, comenzando por "Ay, mi cabeza [manos/ pies] que no me dan” intercalando distintas partes del cuerpo para continuar la canción, moviéndolas o señalándolas y pidiendo que las nombren en mapudungun: "La tengo tiesa como un compás, como un compás, como un compás/ Manteca de sañue le voy a echar, para que se mueva de aquí para allál De aquí para allá, de aquí para allá”. Al mismo tiempo que modela los movimientos e involucra a los niños para que la sigan, les pregunta “¿Y qué quiere decir sañue?”, a lo que algunos alumnos contestan "¡Chancho!”. De tal forma vincula la actividad a la práctica del idioma. Una vez realizada la primera ronda de canciones dedicadas a las distintas partes del cuerpo, la profesora repasa los conceptos cantados en mapudungun (pies: namun; mano: küwï; cuello: pel; cintura: wenteyi; cuerpo: che kalul), señalándolos en su propia persona.

En la clase siguiente, David, un niño que suele tener problemas conductuales y no había mostrado mucho interés durante la clase de la canción sobre el che kalul, revela una actitud diferente frente a la actividad. Mostrando a una investigadora una figura humana recortada y coloreada como el Hombre Araña, David entona la canción "Lonko, lipan, kuwu [...]", apuntando con su dedo índice a cada parte del cuerpo de su "Hombre Araña". Para responder a la pregunta de la investigadora sobre cómo se dice mano $(k u w u)$, inicialmente David vuelve a cantar la canción completa, pero en un segundo momento busca con interés deducir los nombres de las distintas partes del cuerpo sin tener que cantarla desde el comienzo. 
Cabe destacar los distintos momentos que protagoniza David en su vinculación con el recurso canción. Desde un principio David, por iniciativa propia, replica el uso "demostrativo" que hace la profesora de la canción para indicar a la investigadora las partes del cuerpo correspondientes en la figura del Hombre Araña. Sucesivamente, David utiliza autónomamente la canción como artefacto y soporte cognitivo para recobrar la información que le permita responder a las preguntas de la investigadora. Por último, logra conceptualizar los nombres en mapudungun sin hacer uso del medio "canción”. Así, la canción ha cumplido plenamente su función de artefacto mediador en el aprendizaje de David.

\subsubsection{Los segmentos de tiempo y espacio}

Este material lo conformaban segmentos de cartón impresos -con la forma de una tajada de pizza- que pudiesen completar un semicírculo que ilustrara el trayecto y posición del sol durante el día, montados sobre unos rieles de madera en un atril. Completando el conjunto se encontraban modelos bidimensionales de cartón impreso de montañas y de una ruka mapuche, para dar puntos de referencia del movimiento del sol desde las montañas al mar, con la ruka como centro de convergencia de los segmentos aludidos. Es importante señalar que este material fue introducido en el aula sin que la profesora pudiese familiarizarse con él de antemano y diseñar una estrategia para su efectivo aprovechamiento en la clase. Esto explica, en buena medida, la debilidad de la práctica docente en el uso de este recurso.

En la clase en que se utilizó el material, se montó el atril con las montañas y la ruka, y la profesora comenzó a explicar de qué se trataba la representación. Hace referencia a un epew, visto en una clase anterior, acerca del chinke con el fin de que recordaran el papel del antü (sol). A los pocos minutos de iniciada la actividad, los niños pierden la concentración y se generan varias interrupciones, con un variado nivel de reactividad por parte de la profesora. Al cabo de una media hora, el estilo mayormente expositivo de la profesora acerca de un tema complejo, con un manejo del material exclusivo de su parte, termina por desconectar completamente a buena parte de los niños de la actividad.

El mismo material (aunque ampliado) se utilizó en el segundo año básico, con resultados similares en cuanto a niveles de atención y comprensión. Al parecer, el orden y forma de tratar los contenidos, incluido su nivel de detalle, parecieron apegarse a un modelo rígido, con nula atención al estado de ánimo colectivo y atención del curso. Por otra parte, la pregunta de un niño “¿No vamos a trabajar?”, revela que el trabajo para los estudiantes estaba asociado a alguna forma de actividad directa, en particular asociada al uso y llenado de fichas. En este sentido, la utilización de un material de manera puramente expositiva, en relación con conceptos de un nivel mayor de abstracción resultaron en una combinación ineficiente tanto para el objetivo de transmitir los contenidos, como para generar el interés e involucramiento de la clase.

\subsection{RECURSOS UTILIZADOS POR LOS NIÑOS}

\subsubsection{Fichas}

Todas las clases del Sector Lengua y Cultura contemplan una actividad de trabajo con fichas que, por lo general, se realiza posteriormente a una primera actividad de carácter más expositivo liderada por la profesora. La ficha es una hoja de trabajo individual donde, 
impresas en blanco y negro, están las instrucciones para la realización de una actividad a realizar sobre la misma, sea que se trate de pintar, recortar o escribir. Por lo tanto, la ficha no solamente entrega indicaciones sobre la tarea a realizar, es también el objeto mismo donde ésta se ejecuta. Tal objeto usualmente contiene imágenes y conceptos en mapudungun que son los que se han tratado en la clase y durante la exposición oral de la profesora. En este sentido, la ficha cumpliría la función de reforzar los contenidos y conceptos trabajados ofreciéndoles a los niños la posibilidad de "aplicarlos" de manera autónoma. La primera tarea en el trabajo sobre la ficha es la escritura del nombre en la línea que dice: Tañi üi (mi nombre). Registrar el nombre en la propia hoja de trabajo es particularmente relevante desde el punto de vista académico porque las fichas son la principal evidencia del aprendizaje de los niños. Éstas, por lo tanto, constituyen un doble instrumento de evaluación: del trabajo de los niños y del trabajo de los profesores.

En la clase sobre las partes del cuerpo la profesora entrega una ficha titulada "Che kalul" con una figura humana cuyos miembros están separados como para ser recortados. No introduce el tema de trabajo posiblemente porque ya se ha abordado en la clase anterior y da la instrucción de colorear y recortar las partes del cuerpo. Los niños trabajan concentrados en la tarea. La investigadora recorre los puestos preguntando a los niños qué están haciendo pero los niños o no conocen la respuesta o responden equivocadamente.

Frecuentemente el trabajo de las fichas absorbe -por lo menos durante un tiempo- la concentración de los niños quienes, por lo general, reciben positivamente la instrucción de colorear. Por el entusiasmo que genera esta actividad en los niños, todas las fichas presentadas la incluyen entre sus tareas si no como tarea principal. El supuesto pareciera ser que, a medida que pintan las imágenes, los niños se familiarizan conceptualmente con lo que éstas representan, reforzando los contenidos asociados que se han trabajado previamente. Al mismo tiempo, la concentración sobre las palabras en mapudungun escritas en la hoja lograda por medio de la actividad de pintura sobre ellas, debiera, según la misma lógica, favorecer que esas palabras "se inscriban en la mente" de los niños. Sin embargo, tal supuesto se revela falaz porque la actividad manual de los niños sobre las fichas muchas veces no muestra tener conexión alguna con los conocimientos que se espera que ellos adquieran.

Como muestra la clase sobre la ficha del Che kalul, los niños colorean y recortan sin tener claro el sentido de la actividad ni preocuparse si manejan los conceptos implicados en ella. La posibilidad que brindan las fichas de manipular objetos -lápices de colores, tijeras, pegamento y papel- las convierte en una instancia motivadora y placentera para los niños, pero desvinculada de los aprendizajes que se espera lograr con ellas. Evidentemente, la actividad manual sobre las imágenes y palabras no cumple con los objetivos de aprendizaje pero, al tener un componente recreativo y motivacional, contribuye significativamente a mantener el orden y la disciplina, un "bien" escaso en las clases del Sector del primer año.

\subsubsection{Rompecabezas}

Otro de los materiales utilizados directamente por los estudiantes fue una combinación de tarjetas con palabras en mapudungun y el rompecabezas de una figura humana. En términos globales, la idea de la actividad fue entregar un set de palabras en mapudungun identificando partes del cuerpo (brazo, cabeza, cuello, etc.). Sin embargo, cada palabra estaba constituida por más de una tarjeta, cada una conteniendo las sílabas de la palabra, las 
que había que ordenar correctamente para obtener el concepto indicado. Así, la actividad consistió en un desafío para grupos de trabajo, a los que se entregaba por rondas un set de tarjetas -igual para cada grupo-, para que las ordenaran formando la palabra buscada en mapudungun.

Una vez reconstruida la palabra en el orden correcto de las sílabas, los equipos debían decir en voz alta fey müten para indicar que habían terminado, y podían decirle a la profesora cuál era la palabra y su traducción. Si el grupo resolvía correctamente la tarea, la profesora les entregaba una parte del rompecabezas representando el concepto, para de esta manera ir ensamblando las partes y terminar formando la figura humana completa.

En el desarrollo de la actividad, tanto en primer como en segundo año, se presentaron aspectos positivos comunes así como fallas comunes. El hecho de presentarse como un juego y una competencia entre grupos hizo que los estudiantes se vincularan de manera entusiasta y bastante explícita. Sin embargo, el articulado de la actividad hizo que los objetivos fueran difíciles de cumplir. Uno de los principales problemas fue que los conceptos se presentaron en el mismo orden, es decir, todos los grupos debían ordenar el mismo concepto. Esto hizo que comenzaran a copiarse entre grupos, al escuchar los intercambios vecinos. Por otra parte, el procedimiento más típico e inicial era por ensayo y error, y no tratando de recordar cuál era la palabra buscada. Así, ordenaban las dos o tres tarjetas de una manera, gritaban fey müten, esperaban a que la profesora confirmara o rechazara el orden y probaban con otro, de manera mecánica.

En suma, la profesora sí logró generar un espacio de repaso de los conceptos, revisando las partes del cuerpo en voz alta, con el modelo humano-rompecabezas y consigo misma modelando. Sin embargo, sigue siendo central el hecho de que la actividad misma no pareció cumplir con los objetivos planteados. Aún más, se presenta el problema evidente de atribuir conocimiento y manejo de conceptos a los estudiantes con base en evidencia insuficiente y resultados de procesos de ensayo y error mecánicos.

\subsubsection{Rakin awar}

Este material está basado en un juego tradicional mapuche llamado awarkuzen, un juego de mesa de oposición con carácter azaroso (Cortés, 2002), el cual fue adaptado y reinterpretado para que los niños pudiesen aprender el conteo de números en lengua mapudungun mediante un artefacto rico en simbolismo cultural. El elemento central que compone el rakin awar es un pequeño tapiz de lana de oveja tejido a telar y sujeto entre dos listones de madera con diez pequeños agujeros. En ellos se van colocando los porotos secos utilizados para marcar los puntos de los adversarios (uno para cada listón) durante el juego. El juego consiste en tirar sobre el tapiz, por turnos, diez habas (awar) secas y pintadas de rojo por un lado. Se obtiene un punto si al tirarlas aparecen cinco o más del lado rojo y dos puntos si son las diez. Los niños después de tirar las habas tienen que contar en mapudungun las que aparecen rojas.

La introducción del rakin awar representó un significativo quiebre con el usual esquema de clases en el Sector Lengua Indígena. La primera vez que se implementa con el primer año, la profesora cambia la ubicación de las mesas en la clase y dispone a los niños uno frente a otro para que puedan jugar en pareja. Las bolsitas del juego se reparten en pareja y mientras los niños se dedican a explorar los curiosos objetos contenidos, la profesora introduce el juego explicando que tiene su origen en el juego tradicional: "Awar 
es haba y kuzen juego". Entrega a cada pareja una hoja donde aparecen escritos (en letras) con correspondientes imágenes los primeros diez números en mapudungun como un apoyo para el conteo en lengua.

Sentados en su puesto y concentrados en el juego, los niños muestran entusiasmo, exultan de alegría cuando marcan un punto o cuando están en ventaja de varios puntos. Al cabo de pocos minutos varias parejas cuentan rápidamente en mapudungun y sin mirar la hoja de apoyo. Llama la atención la pareja donde hay un niño diagnosticado como autista, llamado Pedro. Su compañera Elena empieza a jugar mirando la ficha que la profesora entregó como guía. Pedro recibe apoyo de un miembro del equipo de investigadores para seguir el juego y empieza contando en castellano. Después de pocos minutos Elena ya está contando fluidamente en mapudungun sin mirar la ficha mientras Pedro está haciendo el intento. La compañera le ofrece un andamiaje válido para que él pueda contar de manera más autónoma aunque cometiendo algún error. Así Pedro empieza: "keñi [en lugar de kiñe], epu, kula [...]".

Contrariamente a los segmentos de tiempo y espacio, este segundo recurso diseñado por el equipo de investigadores ha tenido una muy buena acogida de parte de los niños, incluso entre los que tienen mayor dificultad en el plano académico, sea por problemas conductuales o por trastornos del desarrollo, como es el caso del niño autista. El formato de juego y el factor de la competición o desafío, la posibilidad de manipular objetos concretos y reales, como son las habas y los porotos, la accesibilidad del desafío planteado y la existencia de reglas simples y claras permitieron estimular y mantener un alto nivel de motivación incluso en los alumnos normalmente más desencantados de las actividades escolares, junto al uso de materialidades que evocan el arraigo cultural del artefacto (lana cruda, madera y legumbres secas).

Sin embargo, nos parece oportuno rescatar de esta acotada experiencia no tanto el aspecto motivacional, fácilmente predecible por las características señaladas, sino como éste se conjuga con el cumplimiento de los objetivos de aprendizaje para todos los niños, sin importar su nivel de habilidades o conocimientos previos. Es decir, contrariamente a otras "actividades lúdicas" implementadas por la profesora, al involucramiento físico y emocional de parte de los niños correspondió un proceso de cambio en el manejo de la habilidad de contar en lengua mapuche. Nos parece relevante poner en evidencia que el ejercicio de conteo ocurre durante la actividad como un requisito central para el logro de la misma; ésta era, para los niños, una tarea necesaria para verificar si habían logrado los puntos necesarios para ganarse uno o dos porotos y esa expectativa era fundamental para acelerar la adquisición de los conceptos en mapudungun, puesto que la regla era que sólo se podían contar las habas en mapudungun para hacer valer los propios puntos.

\section{ANÁLISIS DE LOS RECURSOS EDUCATIVOS IMPLEMENTADOS}

Antes de entrar en un examen más acabado de los recursos educativos implementados en el SLI, es oportuno aclarar que por las características y alcances de este estudio, de carácter cualitativo y etnográfico, el análisis de su potencial en términos de "artefactos mediadores" del pensamiento o recursos para el aprendizaje se basa principalmente en la motivación intrínseca que pudo detectarse en los aprendices al interactuar con esos artefactos. Sin ser el objetivo de este trabajo explorar los multifacéticos procesos psicológicos que explican la dedicación y persistencia de los niños en las actividades (Mitchell, 1982), por medio de 
la observación etnográfica se ha focalizado la atención en aquellas claves conductuales que daban cuenta del interés e involucramiento de los niños en las actividades propuestas. Es en este sentido genérico que hemos abordado la motivación, es decir, como un deseo o inclinación hacia alguna actividad (Buehl \& Alexander, 2009), y como la "energía" que los estudiantes ponen en su ejecución (Wentzel \& Wigfield, 2009).

Centrándonos en los recursos y las actividades didácticas, la primera pregunta que hemos intentado responder es la siguiente: ¿Qué hace que éstos sean intrínsecamente motivadores para los niños? En línea con los estudios de corte más psicológico sobre motivación, nos hemos basado en la premisa ampliamente aceptada de que la motivación es un factor clave para el aprendizaje (Bransford, Brown \& Cocking, 1999; Pintrich, 2003; Pintrich, Marx \& Boyle, 1993), y que la intervención educativa en el plano motivacional es fundamental para el mejoramiento del aprendizaje académico (Wentzel \& Wigfield, 2009). Por otra parte, nuestro análisis también tomó en cuenta la respuesta cognitiva observable al estímulo ofrecido, es decir, un primer nivel de aprendizaje en relación al manejo de contenidos o habilidades intencionado con la actividad propuesta. En este sentido, la segunda pregunta que abordamos es: ¿Qué convierte a las actividades o los recursos educativos intrínsecamente motivadores en efectivos en términos de aprendizaje? La perspectiva del aprendizaje situado (Lave \& Wenger, 1991; Rogoff, 1984) guía nuestra respuesta en este ámbito.

En términos generales, los recursos que hemos categorizado como "utilizados por los alumnos", generaron un nivel más alto de motivación. En la medida que los niños tenían un rol menos pasivo y podían involucrarse corporalmente y de manera más integral a las actividades, manifestaron un mayor interés en llevarlas a cabo y un mayor nivel de atención. Es el caso de los recursos descritos como fichas (especialmente las que implicaban colorear y pintar), rompecabezas, rakin awar y canciones (cuando no tenían un carácter meramente demostrativo).

Sin embargo, si una participación más plena de parte de los niños en las actividades, donde más de un sentido está involucrado -conforme a nuestra definición de recurso educativo efectivo- impacta positivamente su nivel de motivación, no necesariamente se asocia a ésta el cumplimiento del objetivo de aprendizaje. Esto porque en la mayoría de los casos, la participación "manual" de los niños (en inglés se describen como "hands on" activities) está desvinculada de las operaciones conceptuales que dan sentido a la experiencia en el ámbito académico. En otras palabras, pintar, recortar, trabajar con plastilina y armar un rompecabezas son actividades que los niños emprenden con relativo entusiasmo y que, por lo tanto, ayudan a mantener un nivel más o menos aceptable de orden y disciplina en la sala de clase.

No obstante, es cuestionable que a esa concentración e interés de parte de los niños corresponda algún nivel significativo de aprendizaje de los conceptos en mapudungun relacionados con la actividad. El caso de la tarea de recortar y colorear en la ficha sobre el che kalul es un ejemplo claro de esta disociación: los niños no tienen conocimiento del objeto de su actividad manual, es decir, no saben expresar en mapudungun el concepto que se refiere a lo que colorean, y tampoco pareciera importarles esa información porque no es relevante para llevar a cabo lo que sí les interesa más (colorear y recortar).

Las canciones son un ejemplo de actividad donde existe una integración entre participación corporal y elaboración conceptual. El ritmo, la melodía y el movimiento les ayuda a memorizar algunos términos en mapudungun. Por otra parte, el elemento 
constitutivo de la canción es la verbalización de los conceptos a aprender. Si bien puede resultar algo mecánico en un principio -la conceptualización está "arrastrada" por esos componentes mediadores de apoyo a la memoria-, ocurre en algún momento que los conceptos se recuperan de forma autónoma, tal como muestra el caso de David, quien, interrogado, recobra la información que necesita, desvinculándola de la canción. Este caso muestra la necesidad de planificar un ejercicio posterior a las canciones o a otras actividades similares (en que la cognición es parte integrante de éstas) para desligar la conceptualización de la acción en el marco de la tarea. Posteriormente al rakin awar, la profesora estimula este ejercicio al dirigir preguntas a los niños que ellos tienen que contestar empleando los conceptos aprendidos durante el juego, es decir, los números en mapudungun.

Anteriormente se han señalado algunos factores que hacen del rakin awar una actividad especialmente motivadora para los niños de primero y segundo básico. Pero también se ha puesto en evidencia que, contrariamente a otras actividades, el rakin awar se configura como una actividad lúdica efectiva en términos de aprendizaje, donde a la participación “corporal” y motivación de los niños corresponde el desarrollo de la habilidad lingüística, planteada como objetivo académico. En el contexto del juego, la simple acción de entrenar el conteo y los números en otro idioma se convierte en una actividad significativa, porque va asociada al cumplimiento de un objetivo de alto interés: ganar una competencia. En otras palabras, la cognición opera como una herramienta para enfrentar un desafío real, el que plantea la situación del juego. Estos dos componentes, valor instrumental de la cognición y situación desafiante, desde nuestra perspectiva, convierten a un serious game ${ }^{2}$ como el rakin awar en un ejemplo de aprendizaje situado en el contexto escolar.

A primera vista, esta reflexión puede sonar paradójica porque en la literatura se suele asociar el aprendizaje situado a los procesos cognitivos que se dan en el marco de actividades cotidianas de la vida, como hacer las compras en el supermercado (Lave, Murtaugh \& De La Rocha, 1984), realizar labores no especializadas en una fábrica (Scribner, 1984), o tomar decisiones de política institucional (Mehan, 1984). Los estudios de cognición situada han mostrado que "pensar está intricadamente entrelazado con el contexto del problema a resolver" (Rogoff, 1984: 2) ${ }^{3}$, lo cual explica por qué a veces no logramos recobrar información o mostrar ciertas habilidades fuera de la situación en que normalmente las activamos.

Así, tal como han estudiado Lave, Murtaugh \& De La Rocha (1984), algunas personas muestran habilidades matemáticas durante la compra del supermercado que no aparecen en el contexto de un test de tipo académico. Las representaciones que nos hacemos en nuestro quehacer diario son extremadamente dependientes de la relación con objetos, símbolos y personas en determinadas situaciones, lo que sin embargo no quiere decir que todo conocimiento es específico. La generalización es posible y necesaria para poder aplicar el conocimiento a nuevas situaciones, nos recuerda Rogoff (1984: 3). No obstante la recurrencia, a lo largo de distintas actividades, de ciertos elementos contextuales o ambientales asociados a esas representaciones es un factor clave para mejorar el desempeño en determinadas tareas (Brown, Collins \& Duguid, 1989: 37). Esto también se aplica para el concepto de artefacto mediador del pensamiento, que siempre está funcionando en un

Según Michael y Chen (2006) el término "serious games" está referido a juegos en donde la educación, en sus más variadas formas (aprendizaje, entrenamiento o información), es el objetivo primario por sobre el entretenimiento.

Traducción del texto original. 
contexto determinado. Si su uso no es adecuado vía mediación de otros actores sociales, o no se ajusta al escenario, simplemente pierde pertinencia y, por tanto, efectividad.

Los estudios de cognición situada o aprendizaje situado han ofrecido importantes insumos para repensar el aprendizaje en el contexto escolar. Como todo aprendizaje es situado, el aprendizaje en la escuela no es una excepción, pero en la misma cultura escolar y no en los contextos originales que otorgan significado a lo que se aprende en la escuela. Es decir, ninguno de los contenidos o habilidades que se aprenden a través del curriculum escolar es transmitido de la misma manera en que se aprende cualquier contenido en la vida diaria, o sea, en el marco de una cultura disciplinar dada (ciencias, aritmética, etc.) donde se aprende a utilizarlos de manera apropiada (Brown, Collins \& Duguid, 1989). Por lo mismo, el aprendizaje significativo que ocurre en la escuela no dice relación con las materias del curriculum, sino con prácticas, valores y símbolos que son propios de la dinámica de la vida escolar. Desde la perspectiva del aprendizaje situado, el desafío a nivel pedagógico es diseñar contextos o situaciones que sean lo más cercanas posibles a la vida real, en otras palabras, a contextos y actividades "auténticas" (Brown, Collins \& Duguid, 1989; Herrington \& Oliver, 2000; Sagástegui, 2004). Simular una transacción de compraventa aplicando conocimientos matemáticos o diseñar torres y puentes de papel (Wolff-Michael, 1996) aplicando conocimientos ingenieriles, son ejemplos de actividades de aprendizaje situado en la sala de clase.

Llegados a este punto cabe preguntarse en qué sentido el rakin awar, en cuanto "juego de tablero", puede asemejar una situación de la vida real, tratándose justamente de una actividad que se constituye como una suerte de evasión de aquella: los participantes, quienes asumen roles de contrincantes, realizan acciones que no tendrían ningún sentido en la vida real, como manipular habas pintadas a modo de dados y porotos secos como marcadores de puntaje. La autenticidad del rakin awar, a nuestro juicio, se debe al hecho de que genera una situación per se auténtica, donde el problema o desafío es real y las operaciones cognitivas son funcionales para la consecución del objetivo propio de la actividad.

En este sentido, el rakin awar difiere tanto de las fichas como del rompecabezas, que también plantea una competencia, porque ni en las primeras ni el segundo el desempeño cognitivo de los niños, que es el objetivo académico, es efectivamente necesario para el propósito de la actividad. En el caso del rompecabezas hizo falta una regulación más estratégica de la dinámica del juego que, por ejemplo, impidiera cumplir con el objetivo (el ensamblaje de la tarjeta-concepto) por medio del ensayo y error. Esta regulación, en cambio, estuvo presente en el rakin awar donde se dejó en claro que sólo contando en mapudungun se podía hacer valer la propia ventaja sobre el adversario. Por otro lado, otras características del rakin awar corresponden a aspectos propios del aprendizaje situado, como la interacción social y el andamiaje otorgado por el más experto (Collins, Brown \& Holum, 1991).

Esto se reveló en el caso del niño autista que llevó adelante la tarea de conteo apoyado por su compañera-adversaria. El rompecabezas, por su parte, también presenta aspectos de aprendizaje situado por ser una "actividad lúdica"4. En cuanto juego, el rompecabezas construye un contexto espacio-temporal, una actividad y un objetivo auténticos, además de

\footnotetext{
"Lúdica" se define como esa dimensión del desarrollo humano que se manifiesta en una amplia gama de actividades donde interactúan el placer, el gozo, la creatividad y el conocimiento. Dentro de sus múltiples manifestaciones destaca el juego, actividad que se realiza libremente, sin un fin utilitario, de acuerdo a reglas establecidas previamente por los participantes. Éste se desarrolla fuera del curso habitual de la vida o en una zona de distensión dentro de lo real. En sus formas más estructuradas posee un carácter agonal (de enfrentamiento) donde su fin es la resolución de una tensión o conflicto (Huizinga, 2012).
} 
contemplar la interacción social, el andamiaje y la construcción colectiva de conocimiento, evidentes en la operación conjunta de ensamblaje de la palabra-tarjeta. Sin embargo, está claro que la posibilidad de escuchar la solución del equipo cercano o de encontrarla por ensayo y error limitan el potencial de este juego.

En el caso de los epew, es oportuno detenerse a analizarlo como un caso peculiar de "recurso expositivo" que genera un alto nivel de interés y participación entre los niños. Esto se evidenció en múltiples ocasiones en que mostraron su preferencia por escuchar un cuento antes que "trabajar" en las fichas. Consideramos que para explicar el "éxito" de los epew hay que tomar en cuenta varios elementos: en primer lugar, la accesibilidad y familiaridad del tema, relacionado con animales del entorno cercano de los niños, que son parte de su mundo experiencial; en segundo lugar, el apoyo visual en ilustraciones simples pero suficientemente atractivas para los niños como para estimular su interés e imaginación. No deja de ser una importante contribución, también, el tono recitativo de la profesora al leer el cuento que cautiva la atención de los niños y la capacidad de la profesora de insertar, durante la recitación, referencias a experiencias personales o a situaciones reales, así como de dejar espacio para la participación de los niños.

Además, es oportuno considerar la estructura narrativa del cuento, que lo convierte en un recurso universalmente empleado para la transmisión de conocimiento. El cuento se sitúa también en un plano de "autenticidad" en la medida que reproduce estructuralmente, en términos generales, el cómo nos narramos eventos en la vida cotidiana. Así, según Shore (1996: 58), la narración se asume como una actividad regular, mediante la cual la experiencia es "hablada" para ser provista de sentido. Ésta da lugar también a relatos como productos culturales (p. ej.: como un cuento, una leyenda, una narración más o menos fija) que funcionan como "modelos culturales lingüísticos", proveyendo de contenidos varios.

Sin embargo, las narraciones se encuentran en una relación creativa mutua con el acto de relatar, lo que mantiene a nuestro juicio su carácter "auténtico" a la hora de ser utilizados en planos educativos. En este sentido, debemos replantearnos el supuesto carácter "expositivo" de la narración de cuentos, ya que cada oyente se encuentra en un proceso activo de construcción de representaciones y manipulación de las mismas, que resultan altamente pertinentes y significativas en la medida que provienen de los conocimientos propios y previos del oyente. Así, se genera actividad cognitiva "distribuida" en un espacio mental compartido (Cole \& Engeström, 1997: 4-9; Shore, 1996: 75; Wertsch, 1991: 14), en particular si el educador permite los comentarios y preguntas de los niños mientras la actividad se desarrolla, intencionando además la contextualización socio-cultural del relato. Por último, si a esto se suman los elementos que divergen de la narración regular, como personajes fantásticos o animales antropomorfizados, contenidos metafóricos, etc., los cuentos se vuelven artefactos culturales particularmente atractivos para los niños.

\section{CONCLUSIONES}

De esta manera, el estudio del caso de implementación del Sector Lengua Indígena en una escuela de la comuna de Pucón permite reflexionar sobre el potencial del SLI de adquirir un carácter único y de obtener un impacto pedagógico diferente de las otras asignaturas. Esto último se da por estar intrínsecamente asociado, por lo menos en principio, al sentido de identidad y vivencia de los niños y a un repertorio de conocimientos no pertenecientes a 
la cultura oficial y mayoritaria. Esta potencialidad es percibida por la profesora de nuestro estudio de caso, quien, personalmente motivada por el desafío y la oportunidad de enseñar la cultura propia, busca formas alternativas de transmitir los contenidos para que el SLI pueda tener una buena acogida de parte de los niños.

A partir de este estudio consideramos que, si bien la escasez de recursos pedagógicos puede ser problemática para la labor de los profesores, ésta se configura también como una oportunidad por el estímulo que ofrece a buscar y crear nuevas metodologías y medios poco comunes en las prácticas de enseñanza de las asignaturas tradicionales. Por lo mismo, consideramos que esta oportunidad debiera ser tomada por los organismos estatales interesados en la implementación del Sector Lengua Indígena (CONADI, MINEDUC, Municipalidades) y por las Universidades, tanto en la formación docente como para la investigación e innovación educativa.

En este artículo nos centramos en el papel de los recursos educativos en la implementación del SLI, y en el aprendizaje de los contenidos propuestos en ese marco. En nuestra aproximación a los recursos se articulan tres perspectivas. La primera, que deriva de los estudios socio-culturales originados en el pensamiento de Vygotsky, se centra en el rol de la mediación social y cultural para el desarrollo cognitivo (Rogoff, 2003) y, a su vez, en el rol de los artefactos de valor simbólico e instrumental para esa mediación (Holland \& Valsiner, 1988). Desde esta perspectiva, consideramos los recursos educativos como artefactos mediadores del pensamiento (Acosta-Iriqui y Esteban, 2010), cuyo significado, valor e impacto es altamente dependiente del contexto y del uso, razón por la cual es imposible aislar el estudio de los recursos educativos de su implementación en un determinado contexto y bajo ciertas circunstancias.

Por otra parte, introducimos una visión de recurso educativo según la cual éste se configura como medio que materializa el diseño de una experiencia. La efectividad del recurso educativo como mediador para el aprendizaje depende de la calidad de esa experiencia. A nuestro modo de ver, ésta, idealmente, tiene que involucrar más de un sentido de percepción, plantear desafíos reales, promover la interacción y colaboración entre pares, y evocar elementos del entorno cercano del niño. El juego del rakin awar que fue diseñado e introducido por los investigadores en las clases del SLI responde a estas características. Esta definición de recurso está en línea con una visión del aprendizaje como situado, es decir, como componente integrante de una situación de desafío auténtica para el aprendiz (Lave \& Wenger, 1991; Rogoff, 1984).

Analizando los recursos educativos implementados y las actividades realizadas desde esta perspectiva, es posible destacar que la creación de un contexto situacional, y la funcionalidad del manejo del artefacto respecto al cumplimiento del objetivo de la actividad, son aspectos claves para el logro de la misma en términos de aprendizaje. Estos dos factores, junto con el andamiaje provisto por la interacción con la profesora y con los pares, convierten a las actividades lúdicas y, en particular, al juego del rakin awar, en recursos educacionales efectivos, donde la cognición, es decir, la elaboración conceptual que es el objetivo de aprendizaje, se conjuga con la motivación de los niños para realizar la actividad. En otras palabras, el juego, pese a presentar una situación ficticia, es un ejemplo de aprendizaje situado al instaurar una situación de desafío que es auténtica y real. Con estas consideraciones no sólo nos sumamos a los estudios que apuntan a valorar el juego como herramienta pedagógica (ver Ortega, 2003a, 2003b, 1999, 1992), sino esperamos ofrecer insumos para el diseño y la planificación de actividades didácticas especialmente para el Sector Lengua Indígena. 


\section{REFERENCIAS BIBLIOGRÁFICAS}

Acosta-Iriqui, J. y Esteban, M. (2010). Geografías psicológicas de los recursos educativos. Revista Educación y Desarrollo Social, vol. 4, n. 2, 119-129.

Bartolomé, A. (2000). Hipertextos, hipermedia y multimedia: Configuración técnica, principios para su diseño y aplicaciones didácticas. En J. Cabero (Ed.), Medios audiovisuales y nuevas tecnologías para la formación del siglo XXI (pp. 127-148). Murcia: DM.

Bransford, J., Brown, A. \& Cocking, R. (1999). How People Learn: Brain, Mind, Experience, and School. Washington DC: National Academy Press.

Brown, J., Collins, A. \& Duguid, P. (1989). Situated Cognition and the Culture of Learning. Educational Researcher, vol. 28, n. 1, 32-42.

Buehl, M. \& Alexander, P. (2009). Beliefs about Learning in Academic Domain. In K. Wentzel \& A. Wigfield (Eds.), Handbook of Motivation at School (pp. 479-501). New York \& Oxon: Routledge.

Carrasco, J. y Basterretche, J. (1998). Técnicas y recursos para motivar a los alumnos. Madrid: RIALP.

Cole, M. \& Engeström, Y. (1997). A Cultural Historical Approach to Distributed Cognition. In G. Salomon (Ed.), Distributed Cognitions. Psychological and Educational Considerations (pp. 1-46). New York: Cambridge University Press.

Collins, A., Brown, J. \& Holum, A. (1991). Cognitive Apprenticeship: Making Thinking Visible. American Educator, vol. 6, n. 11, 38-46.

Coriat, M. (1997). Materiales, recursos y actividades: Un panorama. En L. Rico (Ed.), La educación matemática en la enseñanza secundaria (pp. 155-178). Barcelona: Horsori.

Corrales, M. y Sierras, M. (2012). Diseño de medios y recursos didácticos. Málaga: Innovación y Cualificación.

Cortés, E. (2002). Jeux mapuche: Description et analyse des practiques ludiques d'une société indigène du Chili. Tesis doctoral, Université René Descartes, París, Francia.

Csikszentmihalyi, M. (1998). Aprender a fluir. Barcelona: Kairós.

Dittborn, A., Fuentes, L., Rivas, H., Canales, A. y Gutiérrez, P. (2008). El concepto de recurso educativo en la Sede Regional Villarrica de la Pontificia Universidad Católica de Chile. Informe de Proyecto. Fondo Interno UC Villarrica.

Esteban, M. y Ratner, C. (2010). Historia, conceptos fundacionales y perspectivas contemporáneas en psicología cultural. Revista de Historia de la Psicología, vol. 31, n. 2-3, 117-136.

Gimeno Sacristán, J. (1990). La pedagogía por objetivos: Obsesión por la eficiencia. Madrid: Morata.

Good, C. V. (1973). Dictionary of Education (3 ${ }^{\text {rd }}$ Ed.). New York: McGraw-Hill.

Herrington, J. \& Oliver, R. (2000). An Instructional Design Framework for Authentic Learning Environments. ETR\&D, vol. 48, n. 3, 23-48.

Holland, D. \& Valsiner, J. (1988). Cognition, Symbols, and Vygotsky's Developmental Psychology. Ethos, vol. 16, n. 3, 247-272.

Huizinga, J. (2012). Homo Ludens. Buenos Aires: Alianza Editorial.

Lave, J. \& Wenger, E. (1991). Situated Learning: Legitimate Peripheral Participation. New York: Cambridge University Press.

Lave, J., Murtaugh, M. \& De La Rocha, O. (1984). The Dialectic of Arithmetic in Grocery Shopping. In B. Rogoff \& J. Lave (Eds.), Everyday Cognition. Its Development in Social Context (pp. 67-93). Cambridge: Harvard University Press.

Loncon, E. (2010). Derechos educativos y lingüísticos de los pueblos indígenas de Chile. Revista Isees, n. 7, 79-94.

Luna, L. (2012). Ser etnógrafo de escuelas: Desafíos y oportunidades de hacer etnografía en una institución peculiar. Actas del Tercer Congreso Latinoamericano de Antropología ALA 2012. Santiago, Chile. ISBN 978-956-19-0779-9. 
.e Hirmas, C. (2005). Enfoques curriculares de educación intercultural en Chile: Análisis para una propuesta de convivencia. En M. A Rodríguez (Ed.), Foro de Educación, Ciudadanía e Interculturalidad. Memoria del I Foro Latinoamericano de Políticas Públicas en Educación (pp. 123-138). Cuetzalán del Progreso, Puebla: SEP Coordinación General de Educación Intercultural y Bilingüe; Instituto de Desarrollo, Evaluación Educativa e Investigación A. C.; FLAPE; Observatorio Ciudadano de la Educación.

Markus, H. \& Hamedani, M. (2007). Sociocultural Psychology. The Dynamic Interdependence among Self Systems and Social Systems. In S. Kitayama \& D. Cohen (Eds.), Handbook of Cultural Psychology (pp. 3-39). New York: The Guilford Press.

Mehan, H. (1984). Institutional Decision-Making. In B. Rogoff \& J. Lave (Eds.), Everyday Cognition: Its Development in Social Context (pp. 41-66). Cambridge: Harvard University Press.

Michael, D. \& Chen, S. (2006). Serious Games: Games that Educate, Train and Inform. Boston: Thomson.

MINEDUC. (2012). Educación para preservar nuestra diversidad cultural. Desafíos de implementación del Sector Lengua Indígena en Chile. Santiago: Maval.

MINEDUC. (2009). Marco Curricular Lengua Indígena $1^{\circ}$ a $6^{\circ}$ Básico. Recuperado de http:// www.mineduc.cl/index5_int.php?id_portal $=47 \&$ id_contenido $=17116 \&$ id_seccion $=3264 \& \mathrm{c}=1$, consultado en mayo de 2013.

Mitchell, T. (1982). Motivation: New Directions for Theory, Research, and Practice. The Academy of Management Review, vol. 7, n. 1, 80-88.

Ortega, R. (2003a). Play, Activity and Thought: Reflections on Piaget's and Vygotski's Theories. In D. Lytle (Ed.), Play and Educational Theory and Practice (pp. 99-115). Westport, CT: Praeger. (2003b). El juego: La experiencia de aprender jugando. En J. Gallego y E. Fernández (Eds.), Enciclopedia de Educación Infantil, I (pp. 765-787). Málaga: Aljibe.

Ortega, R. (1999). Crecer y aprender. Madrid: Aprendizaje Visor.

Ortega, R. (1992). El juego infantil y la construcción social del conocimiento. Sevilla: Alfar.

Ossana, E., Bargellini, E. y Laurino, E. (1984). El material didáctico en la enseñanza de la historia. Buenos Aires: El Ateneo.

Pintrich, P. (2003). A Motivational Science Perspective on the Role of Student Motivation in Learning and Teaching Contexts. Journal of Educational Psychology, vol. 95, n. 4, 667-686.

., Marx, R. \& Boyle, R. (1993). Beyond "Cold" Conceptual Change: The Role of Motivational Beliefs and Classroom Contextual Factors in the Process of Conceptual Change. Review of Educational Research, vol. 63, n. 2, 167-199.

Rogoff, B. (2003). The Cultural Nature of Human Development. New York: Oxford University Press.

(1984). Introduction: Thinking and Learning in Social Context. In B. Rogoff \& J. Lave (Eds.), Everyday Cognition. Its Development in Social Context (pp. 1-8). Cambridge: Harvard University Press.

Sagástegui, D. (2004). Una propuesta por la cultura: El aprendizaje situado. Sinéctica, n. 24, 30-39.

Scribner, S. (1984). Studying Working Intelligence. In B. Rogoff \& J. Lave (Eds.), Everyday Cognition. Its Development in Social Context (pp. 9-40). Cambridge: Harvard University Press.

Shore, B. (1996). Culture in Mind. Cognition, Culture and the Problem of Meaning. New York: Oxford University Press.

Szendrei, J. (1996). Concrete Materials in the Classroom. In A. J. Bishop, K. Clements, C. Keitel, J. Kilpatrick \& C. Laborde (Eds.), International Handbook of Mathematics Education (pp. 411-434). Netherlands: Kluwer Academic Publishers.

Wentzel, K. \& Wigfield, A. (2009). Introduction. In K. Wentzel \& A. Wigfield (Eds.), Handbook of Motivation at School (pp. 1-8). New York \& Oxon: Routledge.

Wertsch, J. (1991). Voices of the Mind: A Sociocultural Approach to Mediated Action. Cambridge: 
Estudios Pedagógicos XL, N²: 221-240, 2014

APRENDER LENGUA Y CULTURA MAPUCHE EN LA ESCUELA: ESTUDIO DE CASO DE LA IMPLEMENTACIÓN DEL NUEVO SECTOR DE APRENDIZAJE LENGUA INDÍGENA DESDE UN ANÁLISIS DE "RECURSOS EDUCATIVOS"

Harvard University Press.

Wolff-Michael, R. (1996). Art and Artifact of Children's Designing: A Situated Cognition Perspective. The Journal of the Learning Sciences, vol. 5, n. 2, 129-166.

Zabala, A. y Arnau, L. (2007). 11 ideas clave. Cómo aprender y enseñar competencias. Barcelona: Graó. 